\title{
SOLITONS AND LONG JOSEPHSON JUNCTIONS
}

\author{
R. D. Parmentier \\ Department of Physics \\ University of Salerno \\ I-84081 Baronissi (SA) \\ Italy
}

$\dagger$ This paper is dedicated to

the memory of Gianfranco Paternò.

\begin{abstract}
Magnetic flux quanta, of value $h / 2 e$, in long Josephson junctions behave as (quasi) solitons. Fluxon dynamical states are well described by a perturbed sine-Gordon equation model, with boundary conditions determined by the junction geometry and by externally applied magnetic fields, and they give rise to readily measurable physical phenomena, such as step structure in current-voltage characteristics and microwave radiation emission. Devices based on fluxon propagation offer potentially interesting applications as oscillators and amplifiers - as well as digital applications, described elsewhere in this volume - in high performance integrated superconductive circuits.
\end{abstract}

\section{Introduction}

The combination of 'solitons' and 'long Josephson junctions' brings together the interests of a broad and varied mix of mathematicians, physicists and device engineers. Although the underlying mathematical ideas had been around for some time, the term 'soliton' itself was coined in 1965 by Zabusky and Kruskal [1], just three years after the original work by Josephson [2]. It required some time, however, for the practical importance of the soliton concept to be fully appreciated by the applied science community - certainly more time than was required to absorb the significance of Josephson's work. During the late 1960's and early 1970's 'solitons' and 'long Josephson junctions' began to attract increasing research attention. Starting from key papers by Kulik [3], Scott [4], and Fulton and Dynes [5] (I warn the reader that my literature citations are subjective, partial and incomplete), the literature 
gradually developed, bringing about an improved level of understanding of the basic dynamics of solitons in long junctions. A landmark paper that was particularly influential in focusing interest on the significance of solitons in physical systems was Scott et al. [6]. About the same time, a number of people (see, e.g., Fulton et al. [7]) began to realize that solitons in long junctions offer an exciting potential for practical device applications. In the intervening years research in the field has continued unabated, both 'basic' research, with the development of refined approximate analytical tools and an ever more detailed exploration of 'exotic' topics such as chaos, and 'applied' research, with the development of novel devices such as phase locked arrays of oscillators and the vortex flow transistor.

In this present review my essential task is to attempt an up-date of material presented in two previous reviews, one written by me in 1978 [8] and the other by Niels Pedersen in 1986 [9]. To make this present chapter reasonably coherent and self-consistent it will be necessary to repeat a certain amount of material already presented in these two previous reviews; my main emphasis, however, will be on material that has appeared later.

\section{Device Geometries and Models}

\subsection{GEOMETRIES}

By 'long Josephson junction' we mean simply one that has one dimension, along, say, the $x$-coordinate, long with respect to the Josephson penetration length, $\lambda_{J}$ (see, e.g., Bruynseraede et al. [10] for a concise description of the fundamentals of Josephson tunneling; for a more detailed description see Barone and Paternò [11]), and the other dimension, along, say, the $y$ coordinate, short with respect to $\lambda_{J}$. One might well ask why we choose to focus attention on what is, after all, a rather particular geometrical configuration. I suspect that the true answer here is more a question of analytical convenience than it is of physical importance: a 'small' Josephson junction is described mathematically by an ordinary differential equation (ode); a 'long' junction is described by a partial differential equation (pde) in one space dimension and time; a 'large' (in both the $x$ - and $y$ - dimensions) junction is described by a pde in two space dimensions and time. A $(2+1)$-dimensional pde is a much more complicated object than is a $(1+1)$-dimensional pde.

Long Josephson junctions can be constructed in different geometries, and the junction geometry has a significant effect on the junction dynamics. The most extensively studied geometries are the overlap geometry, the inline geometry and the annular geometry [9]. The first two are somewhat similar in that they both consist of finite-length, quasi-one-dimensional strips; the essential difference between them is the manner in which the bias current is applied to them: in the overlap geometry the current is applied perpendicu- 
lar to the long dimension of the junction, whereas in the inline geometry it is applied parallel to the long dimension. In fact, overlap and inline are idealized limiting cases; real physical finite-length junctions are almost always some combination of the overlap and the inline geometries [9]. The annular geometry, instead, is qualitatively different, consisting of a strip closed upon itself in an annulus. Although somewhat more difficult to construct and control from the point of view of fabrication technology, the annular geometry junction offers the interesting possibility of studying soliton dynamics in the absence of boundary reflection effects.

\subsection{MATHEMATICAL MODELS}

The electrodynamics of a Josephson junction is, in general, described by a $(2+1)$-dimensional pde for the phase difference $\phi$ between the junction electrodes. In normalized form this equation can be written [12]

$$
\phi_{x x}+\phi_{y y}-\phi_{t t}-\sin \phi=\alpha \phi_{t}-\beta\left(\phi_{x x t}+\phi_{y y t}\right)
$$

(we ignore here the so-called $\cos \phi$ term). Here, $x$ and $y$ are the spatial coordinates normalized to $\lambda_{J}, t$ is time normalized to the inverse of the Josephson angular plasma frequency $\omega_{J}\left(\lambda_{J} \omega_{J}=\bar{c}\right.$, the maximum electromagnetic propagation velocity in the junction, called also Swihart velocity), the term in $\alpha$ represents shunt dissipation due to quasi-particle tunneling (normally assumed ohmic, but see Scheuermann and Chi [13] for the effect of a physically more realistic, non-ohmic term), the term in $\beta$ represents dissipation due to the surface resistance of the superconducting junction electrodes [14], and subscripts denote partial derivatives. Details of the normalizations may be found in Ref. [12]. Boundary conditions for Eq. (1), at $x=0, L$ and $y=0, W$, are determined, respectively, by the $y$ - and $x$-components of the magnetic field to which the junction is subjected [11].

For the case of a 'long' junction, i.e., one for which $L \gg 1$ and $W \ll 1$, it is relatively simple to calculate the relevant component of the magnetic field generated by the junction bias current $[11,12]$. For the overlap geometry, assuming a constant (physical) bias current $I_{\text {bias }}$ in the $y$-direction and a spatially uniform, but possibly time-varying, external magnetic field applied also in the $y$-direction, the model reduces to

$$
\phi_{x x}-\phi_{t t}-\sin \phi=\alpha \phi_{t}-\beta \phi_{x x t}-\gamma,
$$

with the boundary conditions

$$
\phi_{x}(0, t)+\beta \phi_{x t}(0, t)=\phi_{x}(L, t)+\beta \phi_{x t}(L, t)=\eta .
$$

Here, $\gamma \equiv I_{\text {bias }} / J_{o} L \lambda_{J}$, where $J_{o}$ is the (physical) maximum pair-current density per unit length in the $x$-direction, and $\eta$ is a normalized measure of 
the $y$-component of the external magnetic field (the normalization is such that, with $\gamma=0, \eta=2$ is the static threshold for magnetic flux entry into the junction). A condition for the validity of the reduction to one dimension is [12]: $\gamma W^{2} / 8 \ll 1$. For the inline geometry the bias enters through the boundary conditions rather than as a term in the pde, since $I_{\text {bias }}$ is now applied in the $x$-direction. Thus, the pde becomes

$$
\phi_{x x}-\phi_{t t}-\sin \phi=\alpha \phi_{t}-\beta \phi_{x x t},
$$

and the boundary conditions become

$$
\begin{aligned}
& \phi_{x}(0, t)+\beta \phi_{x t}(0, t)=-\kappa+\eta, \\
& \phi_{x}(L, t)+\beta \phi_{x t}(L, t)=+\kappa+\eta,
\end{aligned}
$$

where $\kappa \equiv I_{\text {bias }} / 2 J_{o} \lambda_{J}$. Finally, for an annular junction in the absence of an externally applied magnetic field, assuming a spatially uniform bias current density, the governing pde is once again Eq. (2), where $x$ is now to be interpreted as the dimension along the mean circumference of the annulus, and the boundary conditions simply reflect the spatial periodicity of the system:

$$
\phi(x+L, t)=\phi(x, t)+2 \pi n,
$$

where $n$ is an integer (the physical significance of which is the difference between the number of solitons and the number of antisolitons trapped in the junction; it is also known as the 'winding number', for reasons that will become clear during the discussion of the mechanical analog). In the presence of an externally applied magnetic field the right hand side of Eq. (2) must be augmented by the term $\Delta \frac{\partial}{\partial x}(\mathbf{B} \cdot \mathbf{r})$, as discussed by GrønbechJensen [15], where $\mathbf{B}$ is the normalized applied magnetic induction, $\mathbf{r}$ is a unit normal (radial) vector, and $\Delta$ is a coupling constant.

\subsection{MECHANICAL MODELS}

The analogy between the dynamical equation describing a small Josephson junction and that describing a simple plane pendulum subjected to viscous damping and an applied mechanical torque is well known and has been utilized by many authors. The extension of this analogy to the long junction case was quickly recognized and exploited - see, e.g., Scott [4], Fulton [16], and Cirillo et al. [17]; the extension consists of an elastic coupling between adjacent pendula, thus contributing a ' $\phi_{x x}$ ' term to the dynamical equation; a very simple 'pocket calculator' version can easily be constructed by inserting dress-maker's pins into a rubber band at regular intervals. 
In spite of the development of high-speed digital computers in recent years, it is my opinion that the mechanical model of the long junction remains still today an extremely effective stimulus for physical intuition. It renders immediately evident, for example, the nature of the three fundamental classes of excitations on long junctions, viz., plasma oscillations, breathers and kinks - a fact that was recognized, and carefully illustrated, by Fulton [16]. Plasma oscillations, called also 'phonons' or 'radiation' in other contexts, stem from oscillatory motions of the single pendulum (see Fig. 20 of [16]). The lowest-order, $k=0$ ( $k$ is the wave number), mode has all of the pendula in the chain oscillating in a spatially uniform motion; higher-order, $k>0$, modes have a standing wave structure, with one or more spatial nodes along the pendulum chain. For the pure sine-Gordon equation (Eq. (2) with loss and bias terms set to zero), small-amplitude plasma oscillations are characterized by the dispersion relation $\omega^{2}=k^{2}+1$; thus, $\omega=1$ is the lowest possible frequency for such oscillations. Breather oscillations, called also 'bions' in earlier literature, also stem from oscillatory motions of the single pendulum (see Fig. 28 of [16]). They differ from plasma oscillations in that the oscillation amplitude varies with position along the chain, but there are no spatial nodes along the chain. For the pure sine-Gordon system of infinite length, breathers can have frequencies in the range $0<\omega<1$. In contrast, kink oscillations stem from rotary, 'over-the-top', motions of the single pendulum (see Figs. 25 and 26 of [16]); a single isolated kink is just a $2 \pi$ twist in the pendulum chain. Viewing Fulton's careful illustrations also renders intuitive the idea that a breather may be considered to be an oscillatory bound state of a kink and an anti-kink, an idea that has significant consequences for sine-Gordon dynamics. In fact, thoughtful study of Fulton's figures was instrumental in deriving the corresponding exact analytic solutions of the pure sine-Gordon equation on the finite interval, for arbitrary oscillation amplitudes, by Costabile et al. [18].

At this point I should mention that whereas both breathers and kinks of the pure sine-Gordon equation are properly classified as 'solitons' from a strictly mathematical point of view, people who study applications of long Josephson junctions normally reserve the term 'soliton' to mean only kinks. The reason for this is undoubtedly that, due to the dissipative terms - those in $\alpha$ and $\beta$-in the long junction model, plasma oscillations and breathers can exist only as short-lived transients, unless very special forms of driving terms are employed, whereas kinks are highly robust objects that can emerge from a power balance between the $\alpha$ - and $\beta$-dissipative terms and even a simple constant bias current. Kinks are also often called 'fluxons' in the Josephson junction context because each one contains exactly one quantum of magnetic flux, $h / 2 e$ ( $h$ is Planck's constant and $e$ the electron charge); they are also called 'vortices' because the electric current surrounding a fluxon has the form of a vortex. I shall, in the following, use interchangeably - even 
if somewhat improperly - the terms 'soliton', 'kink', 'fluxon', and 'vortex' to refer to these robust, particle-like objects. Finally, the significance of the 'winding number' $n$ in Eq. (7) is now clear: if we introduce $n$ complete $2 \pi$ twists, i.e., 'kinks', into the pendulum chain before closing the two ends into an annulus, the number $n$ is evidently a topological invariant of the system.

\section{Soliton Dynamics}

\subsection{BASIC PHENOMENA}

Let us suppose now that we have introduced-never mind how (for the moment) - a single kink into the interior of a long junction. How will this object behave? The answer evidently depends on the type of junction involved, i.e., the boundary conditions, and on the values of the various parameters of the system; the mechanical model will serve as an aid to the imagination here.

To be specific, suppose that we have an overlap geometry junction with $\alpha \ll 1$ and $\beta \ll 1$, in the absence of an external magnetic field, i.e., $\eta=0$, with a bias current, $0<\gamma<1$. In the absence of the kink, the bias current, i.e., torque, causes a uniform angular offset of the static equilibrium position of the pendulum chain, of value $\phi=\arcsin \gamma$. When the kink is introduced, this applied torque pushes it toward one end of the junction, which end depending on the sense of winding of the kink with respect to that of the applied torque. With $\eta=0$ the two ends of the pendulum chain are free. If the kink arrives at the junction end with enough kinetic energy, the pendulum chain will continue to wind up, i.e., the end pendulum will undergo a rotation of $2 \pi+2 \pi=4 \pi$, and the resulting anti-kink will be pushed toward the other junction end, where the reflection mechanism will repeat. If, on the other hand, the kink's kinetic energy is not sufficient to survive the reflection, i.e., the end pendulum does not receive enough energy to continue winding up, the kink will 'die' at the first end, and, after a transient oscillation, the junction will relax to the spatially uniform static state, $\phi=\arcsin \gamma$. The former case evidently leads to a dynamic steady state, with the kink bouncing resonantly back and forth across the junction, and each pendulum in the chain increasing its angular position by $4 \pi$ after each complete back and forth period of the kink. Since angular velocity, $\phi_{t}$, in the mechanical model corresponds to voltage in the junction, a state with $\left\langle\phi_{t}\right\rangle \neq 0$ should be manifested as structure in the current-voltage characteristic of the junction, a fact recognized by Fulton and Dynes [5], who named such structures 'zero-field steps'. Since a kink behaves in much the same way as does a relativistic particle having a (normalized) limiting velocity equal to unity (physical limiting velocity $=\bar{c}$ ), it follows that these steps tend asymptotically to a constant voltage. An experimental current- 
Fig. 1. Experimental current-voltage characteristic of a long Josephson junction of overlap geometry, showing six zero-field steps. The step index corresponds to the number of fluxons participating in the dynamics.

voltage characteristic of an overlap geometry junction, showing six such zero-field steps, is shown in Fig. 1. The step index corresponds to the number of fluxons participating in the dynamic state; the internal symmetry of a multi-fluxon state can obviously be more or less complicated, but the basic dynamic mechanism remains that of resonant back and forth propagation.

Let us consider now the question, left unanswered above, of how to introduce a fluxon into an experimental junction. To observe zero-field steps, the experimentalist increases the bias current from zero up to the maximum critical value, at which point the junction switches from the zero-voltage state to the gap-voltage state. The equivalent pendulum chain switches from the static state, $\phi=\arcsin \gamma$, to a spatially uniform rotating state when $\phi$ reaches $\pi / 2$. The experimentalist then progressively reduces the bias current, tracing out the McCumber background curve of the characteristic, a piece of which is indicated as 'MCB' in Fig. 1. Correspondingly, the pendulum chain rotates at an ever slower angular velocity. If the bias current is then increased again before the junction returns to the zero-voltage state, it is possible to trace out the zero-field steps, as shown in Fig. 1. The basic mechanism involved was noted by Fulton [16] through observation of the dynamics of a pendulum chain and elaborated analytically only some time later by Costabile et al. [19]: at a sufficiently slow rotation velocity the pendulum chain can develop spontaneously a dynamic instability that results in the 'birth' of one or more kinks.

Suppose now that to the situation described above we add a small dc 
magnetic field, i.e., $0<\eta \ll 1$. This term corresponds to a small torque applied to just the two end pendula of the chain; at one end the $\eta$-torque is in the same sense as the $\gamma$-torque, and at the other end the two torques are opposed. The consequence of adding this term is immediately apparent: at the end where the two torques add, the reflection of fluxons is enhanced, i.e., energy is added to the system; at the other end energy is subtracted from the system, and fluxon reflection is impeded. Increasing gradually the value of $\eta$, this asymmetry between the two junction ends is progressively accentuated until, with an appropriate combination of parameters, a different dynamic steady state configuration can ensue: when the fluxon reaches the energy-subtracting end it 'dies', giving rise to a packet of small-amplitude oscillations (a more or less complicated combination of breathers and plasma waves), which in turn propagates back toward the energy-adding end; at that end, the energy of the packet is sufficient to 'give birth' to a new fluxon, which again propagates toward the energy-substracting end, etc. This is the mechanism responsible for the appearance of 'Fiske steps' in long Josephson junctions [20]. In the dynamic state corresponding to the first zero-field step each pendulum in the chain advances by $4 \pi$ during one complete period; in the dynamic state corresponding to the first Fiske step each pendulum advances by $2 \pi$ during one complete period. This implies that the asymptotic limiting voltage of the first Fiske step is just one-half that of the first zerofield step. Analogously to the situation for zero-field steps, there also exist higher-order Fiske steps having a progressively more complicated internal structure [20].

As the value of $\eta$ is increased still further, yet another dynamic steady state configuration emerges: that of flux flow. As mentioned above, $\eta=2$ is the static threshold for the penetration of magnetic flux into the junction in the absence of a bias current. With $\gamma>0$, this threshold value is reduced (at the energy-adding end); furthermore, a fluxon injected into the junction at this end is accelerated into the interior of the junction by the bias current. When the combination of $\eta$ and $\gamma$ is appropriately above threshold, a dynamic steady state configuration ensues in which fluxons are injected at the energy-adding end as fast as the junction can 'swallow' them; these are accelerated toward the other junction end, where they may be reflected or annihilated, depending on the boundary conditions at that end. A computer simulation illustrating this situation is shown in Fig. 2. The average junction voltage in such a state is proportional to the average number of fluxons inside the junction times their average velocity. Once again, since a fluxon behaves much like a relativistic particle, the current-voltage characteristic tends toward a constant voltage as the average fluxon propagation velocity approaches its limiting value. The resulting steps in the characteristic are commonly called 'flux-flow steps' or 'velocity-matching steps'. The potential interest of flux flow dynamics for practical electronic device applications was 
Fig. 2. Computer simulation showing instantaneous junction voltage in the flux flow regime for parameter values: $L=30, \alpha=0.25, \beta=0.001, \gamma=1.28, \eta=4$. After Zhang [21] (reprinted with permission).

also recognized quite early — see, e.g., Yoshida and Irie [22].

The basic dynamical phenomena in an inline geometry junction are qualitatively quite similar to those in an overlap geometry junction, viz., zero-field steps, Fiske steps, and flux flow. Quantitative differences are due to the difference in the effects of the bias current. Whereas in an overlap junction each pendulum in the chain is subjected to a torque corresponding to $\gamma$, in an inline junction only the two end pendula are subjected to a torque corresponding to $\kappa$, according to Eqs. (5) and (6). In the absence of kinks, each pendulum in an overlap chain is offset in static equilibrium position by the same angle, $\phi=\arcsin \gamma$, whereas the angular offset relaxes toward zero over a physical distance $\sim \lambda_{J}$ (normalized distance $\sim 1$ ) proceeding from the ends toward the interior of an inline chain; the corresponding analytic solution for this latter case was derived by Owen and Scalapino [23] in terms of Jacobian elliptic functions. The most significant consequence of this difference for fluxon dynamics is that a fluxon in an overlap junction feels a constant accelerating force due to $\gamma$ along the entire length of the junction, whereas a fluxon in an inline junction receives an accelerating 'kick' due to $\kappa$ only near the two junction ends.

The situation for an annular geometry junction is quite different because there are no junction ends and because the winding number is fixed for a given dynamical configuration; this means, e.g., that Fiske steps are not present, and that the distinction between zero-field steps and velocitymatching steps tends to be obscured. On the other hand, the possibility of studying fluxon dynamics in the absence of boundary reflection effects is often of considerable interest; in this sense the annular geometry offers a 
'cleaner' environment than does either the overlap or the inline geometries. An interesting feature of fluxon dynamics in annular junctions is that for winding numbers different from zero and in the absence of external magnetic fields there is no zero-voltage current in the current-voltage characteristic of the junction. The reason for this is that as soon as a $\gamma>0$ is applied, any fluxon that is locked into the junction begins to move, which implies that $\left.<\phi_{t}\right\rangle \neq 0$. This situation changes if a small dc magnetic field is applied in the plane of the junction: As mentioned in Sect. 2.2, the presence of an externally applied magnetic field is modelled by adding the term $\Delta \frac{\partial}{\partial x}(\mathbf{B} \cdot \mathbf{r})$ to the right hand side of Eq. 2. If $\mathbf{B}$ is time-independent and spatially homogeneous, this term gives rise to a spatially sinusoidal potential function which can effectively pin fluxon motion $[24,15]$. Very recently, Ustinov et al. [25] have devised an ingenious technique for adding fluxons in a controlled way into an annular junction; this will undoubtedly stimulate further experimental study of this geometry.

\subsection{SOLITON PERTURBATION THEORY}

The only completely general technique (aside from experimental measurement) for studying soliton dynamics in long junctions is that of detailed simulation, i.e., numerical integration of the appropriate pde model. It is frequently convenient, however, to be able to employ approximate techniques to obtain approximate, even though incomplete, information. Soliton perturbation theory furnishes such a technique. Since this topic was discussed quite thoroughly in the 1986 review by Pedersen [9] I shall limit myself here simply to recalling a few salient points. The interested reader should consult McLaughlin and Scott [26] for the mathematical basis of this theory, and the review by Kivshar and Malomed [27] for a detailed discussion of applications to long Josephson junctions.

The basic physical idea involved is that a kink is a highly robust, particlelike object that, even though acted upon by perturbations, tends to maintain its identity; the mathematical foundation underlying this physical fact is that the pure sine-Gordon equation is an exactly integrable system [6]. Accordingly, it is possible to describe the dynamics of this object in terms of its energy, defined as

$$
H=\int\left[\frac{1}{2} \phi_{x}^{2}+\frac{1}{2} \phi_{t}^{2}+(1-\cos \phi)\right] d x
$$

and its momentum, defined as

$$
P=-\int \phi_{x} \phi_{t} d x
$$

The approximation involved in using the soliton perturbation approach derives from the fact that, since we obviously do not know the unknown solution of the perturbed equation, we use instead the known solution of some 
'nearby' unperturbed equation in Eqs. (8) and (9). As a simple illustration of this procedure, let us consider the problem of describing the motion of a single fluxon in an overlap junction, described by Eq. (2), of infinite length. In this case, the time derivative of Eq. (8) may be calculated as

$$
\frac{d H}{d t}=\int_{-\infty}^{+\infty}\left[\gamma \phi_{t}-\alpha \phi_{t}^{2}-\beta \phi_{x t}^{2}\right] d x
$$

We use as a solution ansatz the function

$$
\phi(x, t)=4 \arctan \left[\exp \left(-[x-X(t)] /\left[1-u^{2}(t)\right]^{1 / 2}\right)\right],
$$

which, with $X(t)=u t$ and $u=$ constant, is just the well-known single soliton solution of the pure sine-Gordon equation on the infinite interval. For this unperturbed problem, the energy of the solution described by Eq. (11) may be calculated from Eq. (8) as

$$
H=8 /\left(1-u^{2}\right)^{1 / 2} .
$$

By using the ansatz of Eq.(11), we are implicitly assuming that the essential effect of the perturbing terms - those in $\alpha, \beta$, and $\gamma$ - on the dynamics of the system is to cause a slow modulation of the parameters $X$ and $u$. This modulation is obtained by inserting Eq. (11) into Eq. (10), and using Eq. (12), which gives the result

$$
\frac{d u}{d t}=\frac{\pi \gamma}{4}\left(1-u^{2}\right)^{3 / 2}-\alpha u\left(1-u^{2}\right)-\beta u / 3
$$

and

$$
X(t)=x_{o}+\int_{t_{o}}^{t} u(\tau) d \tau
$$

where $x_{o}$ is the position of the fluxon at time $t_{o}$. In this way, we have reduced the problem from the integration of a pde to the integration of an ode, which provides a substantial computational simplification.

The accuracy of results obtained using the perturbation theory approach depends on how 'close' (in some function-space sense) the solution of the perturbed equation is to the solution of the unperturbed equation used to approximate it in the energy and momentum integrals. A practical example in which the perturbation approach breaks down due to significant discrepancies between the two solutions, caused by the effects of the $\beta$-loss term, was described in detail by Davidson et al. [28]. The only generally valid way to verify the accuracy of perturbation theory results is to compare them $a$ posteriori with pde simulation results. In spite of this inherent weakness, the perturbation theory approach has repeatedly proved to be an extremely useful tool for the study of soliton dynamics. 


\subsection{TIME-DEPENDENT DRIVERS}

So far, we have considered only constant, time-independent bias currents and magnetic fields. In many cases of practical interest, however, the behavior of a long junction in the presence of a time-varying driver is of considerable interest. As a simple illustration of this class of problems, let us consider the inline junction described by Eqs. (4)-(6), subjected to a sinusoidally varying magnetic field, i.e., with $\eta$ in Eqs. (5) and (6) given by $\eta=\eta_{o} \sin (\omega t+\theta)$; this might model a situation in which such a junction is exposed to a microwave field.

The perturbation theory developed in the previous section offers a convenient tool for analyzing this problem. For simplicity we will treat only the case $\beta=0$, which was solved by Salerno et al. [29]; the general case, with $\beta \neq 0$, was solved by Filatrella et al. [30].

For an inline junction with $\beta=0$ the time derivative of Eq. (9) is readily calculated to be

$$
\frac{d P}{d t}=-\alpha P
$$

the general solution of which is obtained by a trivial integration. Moreover, for the unperturbed equation, we have that $P=u H$, with $H$ given by Eq. (12). From these facts the soliton trajectory may be found explicitly from Eq. (14) as

$$
X(t)=x_{o}-\frac{1}{\alpha} \ln \left[\frac{z+\left(z^{2}+1\right)^{1 / 2}}{z_{o}+\left(z_{o}^{2}+1\right)^{1 / 2}}\right],
$$

where $z \equiv P / 8$ and $z_{o} \equiv z\left(t_{o}\right)$.

The other essential ingredient of the analysis is the treatment of fluxon reflections at the boundaries. This problem was solved in the context of the perturbation theory by Levring et al. [31], who showed that during a boundary reflection, due to Eqs. (5) and (6) (with $\beta=0$ ), a fluxon undergoes an energy variation $\Delta E$, given by

$$
\Delta E=4 \pi(\kappa \pm \eta)
$$

where the plus sign is taken at one boundary and the minus sign at the other. This boundary effect can be incorporated into the description of the system dynamics very simply by rewriting Eq. (15) as

$$
\frac{d P}{d t}=-\alpha P+4 \pi \sum_{k=0}^{\infty}\left[\kappa+(-1)^{k+1} \eta_{o} \sin (\omega t+\theta)\right] \delta(X(t)-k L),
$$

in which $\delta()$ is the Dirac delta and $X(t)$ is given by Eq. (16). In writing Eq. (18) we are constructing a mathematical artifice consisting of a periodically 
Fig. 3. Current-voltage characteristic of inline junction with ac magnetic field. Smooth curve: no field; discontinuous curve: with field. Fundamental frequency: $n=m=1$. Parameters: $\alpha=0.05, L=12, \eta_{o}=0.4, \omega=0.225$. Arrow represents switching to the zero-voltage state due to fluxon annhilation.

extended junction structure lying along the positive $x$-axis, between 0 and $+\infty$, in such a way that the back and forth shuttling motion of the fluxon in the physical junction is transformed into a unidirectional, left-to-right motion on the extended structure, with boundary reflection effects taking place at spatial points equal to integer multiples of $L$.

As shown in detail by Salerno et al. [29], the system description can be simplified still further, from the ode of Eq. (18) to an explicit, twodimensional functional map whose variables are $t_{k}$, the time, modulo the period of the applied microwave field, of the $k$ 'th boundary reflection, and $E_{k}$, the fluxon energy at the $k$ 'th reflection. In this way we reduce enormously the computational difficulty of the problem: from integrating the pde system of Eqs. (4)-(6), to integrating the ode of Eq. (18), to iterating a two-dimensional functional map.

The new phenomenon that emerges in this case is that of phase locking: over a certain range of parameters the back and forth motion of the fluxon in the junction becomes locked in phase to the external microwave field; this is most readily manifested as the appearance of a constant-voltage current step in the current-voltage characteristic of the junction, at a voltage $V=\frac{2 n}{m} \omega$, where $\omega$ is the angular frequency of the applied field, and $n$ and $m$ are inte- 
gers, as shown in Fig. 3. Reduction of the system dynamics to a functional map is particularly convenient for studying the phase locking phenomenon inasmuch as one can use standard, well known techniques to study the existence and stability of fixed points of the map, which corresponds directly to studying the existence and stability of phase-locked states of the fluxon dynamics. The salient features that emerge from such an analysis, applied to the system described by Eq. (18), are [29]: (a) Phase-locked steps similar to the one shown in Fig. 3 exist at the fundamental frequency $(n=m=1)$ and at all odd subharmonics $(n=1 ; m=1,3,5, \ldots)$. (b) The height in current of such a step is $2 \eta_{o}$, i.e., equal to the peak-to-peak amplitude of the ac magnetic field; the step is centered around the unperturbed current-voltage characteristic. (c) The stability range in field amplitude for the steps decreases rapidly with increasing subharmonic order, i.e., with increasing $m$. (d) Instability appears first at the center of a step and not, as one might expect, at the extremities. (e) For field amplitude values beyond the stability limit, a Feigenbaum-like bifurcation cascade leading to chaos in the fluxon times of flight across the junction is observed. (f) For appropriate model parameters the phase locking steps can cross the zero-current axis, extending to negative current values; such zero-crossing steps are normally associated with a hysteresis in the step amplitude.

To check the validity of the particle-map formalism used here, it is useful to compare results with those obtained from a full simulation of the original pde model, Eqs. (4)-(6), which has a fairly long and well tested history of accounting for experimental results. Such a comparison shows that the strongest limitation of the particle-map approach stems from the assumption of single fluxon dynamics. Physically, a sufficiently large energy exchange term, Eq. (17), can give rise to the creation of additional fluxons in the junction. Such effects are clearly manifested in pde simulations [32], but they are not contained in the particle-map model; their principal consequence is that the linear growth of the step height in current with $\eta_{o}$ holds only up to a certain limit, beyond which the step height saturates, or even decreases, with increasing field amplitude. Quantitative discrepancies between map and pde results can often be attributed to the fact that a fluxon in the particle-map formalism is a point particle with no spatial extension, whereas a pde fluxon has a non-zero width. Consequently, whereas energy exchange at the junction boundaries occurs instantaneously in the map, the exchange interaction is smeared out over a certain time interval in the pde. This difference tends to disappear for junction lengths $L \gg 1$, but it may be significant for length values of practical interest, especially when subharmonic locking is considered; this problem has been considered in detail, in the context of a perturbation-theory approach, by Grønbech-Jensen [33]. Nonetheless, within its range of validity, the simple map approach predicts results to a truly surprising level of detail and accuracy. 
Fig. 4. Map calculation showing dependence of fluxon time of flight on field amplitude for inline junction with ac magnetic field at third subharmonic. Parameters: $\alpha=0.05, L=10, \kappa=0.1295, \omega=0.3$.

\subsection{CHAOS}

As mentioned briefly in the previous section, solitons in long junctions can also undergo chaotic motion. This is a topic that has attracted a large amount of attention in recent years, and I refer the reader to the literature (see, e.g., Guerrero and Octavio [34], plus references therein) for detailed discussions; I present here only a few particular points. Chaotic effects are observed especially when a junction is subjected to time-varying currents and/or magnetic fields, but the presence of such an ac driver is not strictly necessary to bring about chaos: Soerensen et al. [35] described computer simulations in which a junction that is dc-biased in the Fiske-step regime could, with appropriate parameter values, exhibit chaotically intermittent switching between the dynamic states corresponding to the first and the second Fiske steps; further confirmation and analysis of this phenomenon were subsequently reported by Yeh et al. [36] and by Filatrella et al. [37].

A case in which chaos is brought about by the presence of an ac driver is shown in Fig. 4. This figure shows how the fluxon time of flight, defined as $T_{k} \equiv t_{k}-t_{k-1}$, changes with the amplitude of the applied magnetic field, for an inline junction similar to that discussed in the previous section, using the particle-map formalism [29]; that the phenomenon is real and not just an ar- 
tifact of the map formalism was demonstrated analytically by Malomed [38] and and via pde simulation by Rotoli and Filatrella [39]. The field value at which the first bifurcation in Fig. 4 occurs is just the one for which a stability analysis indicates that the phase-locked state loses stability [29]; the values for the subsequent bifurcations follow quite closely those of a Feigenbaum cascade [40]. An interesting fact that emerges from the map calculations is that throughout the bifurcation region, and even for some distance into the chaotic region, the average junction voltage remains exactly that of the phase-locked state, below the bifurcation tree, even though the fluxon is certainly no longer locked to the driver; this fact may be established by calculating (numerically) the average time of flight in these regions. This means that, in these regions, the complication in the underlying dynamics leaves no signature in the current-voltage characteristic of the junction. Consequently, the only way to reveal such phenomena experimentally would be through a spectral analysis of the radiation emitted by a junction in a state involving fluxon propagation; this poses an interesting challenge to experimentalists.

For most practical electronic device applications the presence of chaotic phenomena is highly undesirable inasmuch as these constitute simply another source of electrical noise, thereby degrading some desired performance characteristic. For this reason, researchers are pressed not only to identify parameter regions where chaos might exist, in order to avoid them, but in fact to devise stratagems to suppress chaos. A step in this direction was recently reported by Salerno [41], who showed, using the particle-map formalism, that the type of chaos described in the preceding paragraph can indeed be suppressed by the addition of a small subharmonic component to the ac magnetic field driving term. Filatrella et al. [42] have provided further credibility for this result via pde simulation; in particular, they showed that, in a situation very similar to that indicated in Fig. 4, the addition of an $m=3$ subharmonic component, i.e., a component whose frequency would correspond to the fundamental for a step at that voltage, approximately one order of magnitude smaller in amplitude than the (true) fundamental, can be sufficient to suppress chaos of the type indicated in Fig. 4.

\section{Electronic Applications}

\subsection{FLUXON OSCILLATORS}

Soliton propagation phenomena in long Josephson junctions, both resonant, back and forth propagation and unidirectional flux flow, give rise naturally to practical applications as electronic oscillators. The reason for this is that a soliton is - amonst other things - an electromagnetic pulse, which, when it impinges on the free end of a junction, gives rise to the emission of a pulse of electromagnetic energy. If the arrival of solitons at the junction end 
is time-periodic, then so is the radiation emission. For typical experimental junctions made with currently available fabrication technologies, i.e., based on low critical temperature materials, this radiation lies somewhere within the microwave to millimeter-wave region of the electromagnetic spectrum. The power emitted is, in absolute terms, rather low: in this context, one microwatt is a 'large' power; on the other hand, existing devices such as SIS mixers, that might conceivably be employed in integrated, all-Josephson millimeter-wave receivers, require not more than this amount of power.

Comparison of the relative merits of oscillators based on the two propagation modes, viz., resonant back and forth propagation and unidirectional flux flow, shows that they have complementary strengths and weaknesses. Single-junction resonant propagation oscillators typically show very narrow output linewidths - about $1 \mathrm{kHz}$ at oscillation frequencies of $\sim 10 \mathrm{GHz}$ have been measured [43] - but observed output powers into an external load have ranged from picowatts up to something less than about a nanowatt - really too small to be practically useful (however, Cirillo et al. [44] have estimated a power of $\sim 100 \mathrm{nW}$ at $75 \mathrm{GHz}$ into a tightly coupled small junction used as a detector). In contrast, for single-junction flux flow oscillators, powers of about a microwatt (into a small-junction detector) at frequencies up to $\sim 400 \mathrm{GHz}$ were obtained quite early [45], and recently much larger valuesfor both power and frequency - have been measured [46]; however, output spectral linewidths for this configuration are considerably larger than for the resonant propagation configuration.

The reason for this relative performance difference between the two configurations is readily understood in an intuitive way: A resonant propagation oscillator is normally operated in zero, or in any case small, external magnetic field. Its oscillation frequency is therefore determined essentially by the junction length and, to a lesser extent, by the dc bias current. This suggests a rather sharply defined frequency, but, since radiation is emitted only during a fluxon reflection from the output end (after a back and forth traversal of the junction), it seems plausible that the output power and fundamental frequency might be relatively low; furthermore, for this same reason, the output voltage waveform of a resonant propagation oscillator consists of a train of fairly sharp spikes, which suggests that output power is delivered into a broad range of harmonics rather than being concentrated at the fundamental frequency. In contrast, the oscillation frequency of a flux flow oscillator depends strongly on the value of the external magnetic field [21], which means that there exists another source of fluctuations that might broaden the output spectral linewidth. However, since fluxons impinge on the output end in a tightly packed, unidirectional train, as shown in Fig. 2, it seems plausible that the output power and fundamental frequency might be relatively high; furthermore, as is also apparent from Fig. 2, the output voltage waveform is quite nearly sinusoidal, which suggests that output 
Fig. 5. Variation of measured output power into an external 50-ohm load from an experimental series-biased, resonant propagation array, with number of active junctions, $n$. Vertical axis normalized to $P_{1}=1$. Points: experimental data; solid curve: parabola $P_{n} / P_{1}=n^{2}$.

power is largely concentrated at the fundamental frequency.

In an effort to boost output power levels and to reduce oscillator linewidths, several groups have begun to explore the idea of employing phaselocked arrays of long-junction oscillators, following the lead of corresponding work done in the context of small-junction oscillators, in large measure by the Stony Brook group [47]. So far, more attention has been dedicated to arrays of resonant propagation oscillators [48-51], in an attempt to raise output powers to practically useful levels, but quite recently the problem of phase locking of a flux flow oscillator has also been addressed [52]. In particular, in the work reported by Monaco et al. [50], phase locking of a controlled number, from one to ten, of series-biased resonant propagation oscillators was obtained at $\sim 10 \mathrm{GHz}$ by capacitively coupling the oscillator array to a high-Q linear distributed resonator. The output power - up to $10 \mathrm{nW}$ in an external, room temperature, 50-ohm load-was found to vary quadratically with the number of locked junctions, as shown in Fig. 5, in accordance with theoretical expectations.

Monaco et al. [50] also showed that the behavior of their device can be described, at least to a first level of approximation, by a simple model in which the physical, distributed resonator is replaced by a lumped, linear 
tank circuit. In this model each junction is coupled to the tank through a capacitance which also comprises a part of the resonator, and the dynamics of the oscillating fluxon in each junction is described by the particle-map approach appropriate to overlap geometry junctions, as outlined above in Sect. 3. To calculate the effect of the junctions on the tank one may assume, consistently with the assumed particle nature of the fluxons, that each fluxon reflecting at the tank boundary can be described as a delta function voltage pulse of weight $4 \pi$. In this way the tank voltage may be calculated analytically as a sum of impulse responses. The effect of the tank on each junction is to modify the junction boundary condition at the tank boundary; specifically, the current fed into each junction by the tank can be calculated knowing the tank voltage and the junction coupling capacitance. The resulting model is formally equivalent to an $(n+2)$-dimensional functional map, in which $n$ is the number of locked junctions. Although not readily tractable analytically, the system dynamics can easily be solved by iterating the map numerically. One salient feature that emerges from such a solution is that the tank voltage amplitude varies linearly with the number of locked junctions, $n$; this implies that the output power from the array varies as $n^{2}$, in agreement with the experimental result shown in Fig. 5.

Although apparently quite promising as an approach to obtain a narrow linewidth oscillator capable of furnishing a reasonable amount of power, the use of arrays of resonant propagation oscillators is subject to some intrinsic limitations regarding the number of junctions that can be locked together [53]; moreover, the practical problems involved in biasing such an array in a simple and controllable manner might well limit the feasibility of this approach. Nonetheless, more work is needed in this direction in order to fully clarify the situation.

Several attempts to design and construct integrated receivers consisting of SIS mixers and long-junction oscillators have been described in the literature. The first of these, to my knowledge, was reported by Crete et al. [54] in 1990. This project consisted of a bow-tie antenna, an SIS mixer, and a 10-junction, resonant propagation oscillator array, designed to operate at $94 \mathrm{GHz}$, a region of considerable interest for radio astronomy applications; no experimental measurements on the complete receiver were reported, however. Zhang et al. [55] presented results on a receiver consisting of an SIS mixer and a long-junction oscillator that could be operated in either the resonant propagation mode or the flux flow mode, depending on the oscillator junction current density - a fabrication parameter subject to direct experimental control. Estimated output powers of $3.2 \mathrm{nW}$ at $106 \mathrm{GHz}$ with the oscillator operating in the resonant propagation mode and $4.6 \mathrm{nW}$ at 261 $\mathrm{GHz}$ with the oscillator operating in the flux flow mode were found for this device. In a successive work Zhang et al. [56] reported measurements on a very similar receiver in which two flux flow oscillators were coupled to the 
same SIS mixer - one oscillator being coupled fairly strongly and the other fairly weakly - so as to measure the output linewidths of the oscillators by mixing them down to a lower intermediate frequency. In this way a composite linewidth of $\sim 2 \mathrm{MHz}$ in the band $280-330 \mathrm{GHz}$ was measured. Moreover, an available power incident on the mixer of $430 \mathrm{nW}$ at $320 \mathrm{GHz}$ was estimated for this device, which is probably more than adequate for optimal mixer performance. Also at 1992 ASC, Koshelets et al. [57] presented results on two receiver designs, both based on niobium trilayer technology. The first of these consisted of a flux flow oscillator coupled to a single smalljunction SIS mixer. For this design, an output power of more than $100 \mathrm{nW}$ at $256 \mathrm{GHz}$ was estimated. The oscillator output linewidth was measured at 82 and $112 \mathrm{GHz}$ by mixing the flux flow oscillator signal with the signal from a backward wave oscillator and analyzing the resulting intermediate frequency spectrum. A value of $\sim 1 \mathrm{MHz}$ was found, which, however, was believed to be an upper limit due to the measurement instrumentation. The second receiver consisted of a flux flow oscillator coupled to a multi-junction SIS mixer array. For this design, a minimum receiver noise temperature of $85 \mathrm{~K}$ at $140 \mathrm{GHz}$ was measured, with an oscillator output power of $\sim 20$ $\mathrm{nW}$.

These results, together with those presented by Andy Smith elsewhere in this volume [58], clearly demonstrate that superconducting technology for the construction of low-noise, integrated millimeter-wave receivers is rapidly approaching maturity.

\subsection{FLUX FLOW AMPLIFIERS}

As mentioned briefly above in Sect. 3.1, that the flux flow mode of propagation offers potential applications for amplification was recognized quite early [22]. In the intervening years, much of the pioneering work in this area has been spearheaded by the group working at the University of Wisconsin in Madison - though it is certainly true that work by the Japanese groups has proceeded continuously. In recent times interest in this application has enjoyed a widespread diffusion, so that today new results are developing rapidly.

The basic idea underlying the operation of a flux flow amplifier is quite readily grasped from the general description of the flux flow phenomenon presented in Sect. 3.1: In the flux flow mode the number of fluxons injected into the junction per unit time depends essentially on the strength of the externally applied magnetic field. Since the average junction voltage is proportional to the product of the number of fluxons in the junction and their average propagation velocity, an increase of magnetic field, at constant bias current, causes an increase of the junction voltage. With an appropriate choice of parameters - in particular, with the junction biased on a velocity 
matching step, and with an appropriate load line - this mechanism can give rise to amplification.

In one of the earliest configurations studied [59], called the "vortex flow transistor' (VFT), the driving magnetic field was furnished by a superconducting control line lying either above or below the junction and electrically insulated from it. In this configuration a time-varying current in the control line causes a time-varying voltage across the junction, so that the basic gain mechanism can be characterized as a transresistance. Properly designed, this device can give significant values of power gain [60].

An alternative procedure for introducing an input signal into such a device is to modulate a current injected directly into the junction counterelectrode [61-63]; this configuration was given the name 'superconducting current injection transistor' (SuperCIT). The basic idea here is that, as suggested by Eqs. (5) and (6), such an injected current plays a rôle in the junction dynamics that is very similar to that played by a magnetic field. In contrast with the VFT, which is normally based on a junction having a low-loss, hysteretic current-voltage characteristic, the SuperCIT is normally based on a junction having a high-loss, non-hysteretic current-voltage characteristic. For this configuration, the device transresistance can be enhanced by constructing the junction with a thin counterelectrode and exploiting the resulting kinetic inductance [64]. However, the SuperCIT has the disadvantage with respect to the VFT of being intrinsically a three-terminal, rather than a four-terminal, device, a fact which leads to obvious grounding problems for the eventual construction of arrays of such devices.

The performance features of both flux flow oscillators and flux flow amplifiers can be enhanced if sharp, i.e., low dynamic-resistance velocity-matching steps can be achieved. A number of attempts to realize such low resistance steps have been based on appropriate tailoring of the junction geometry, in particular, by the addition of 'ears' or 'wings' to the junction structure [21]. An alternative approach, devised by Nevirkovets et al. [65], utilizes a dc edge injection current proportional to the junction bias current, with relative polarities chosen so that the effect of the injection current is to oppose that of the applied magnetic field as regards the injection of fluxons into the junction. The result is a sort of negative feedback which tends to stabilize the number of fluxons in the junction, and hence the average junction voltage. Analytical results [65] and numerical simulations $[65,66]$ suggest that this might prove to be a simple and effective technique for improving device performance.

A related approach to the realization of flux flow amplification has been the so-called 'superconducting flux flow transistor' (SFFT) [67-69]. This consists of an array of weak links - not tunnel junctions - patterned into a single superconducting film, with device input provided by the local magnetic field generated by a separate control line located near one end of the array. Al- 
though the dynamic behavior of weak link arrays has been modelled in terms of a discretized version of Eq. (2) [70], it is not clear that this is entirely correct inasmuch as magnetic flux is carried through such a discrete array by Abrikosov vortices rather than by Josephson vortices (see, e.g., Likharev [71] for a detailed discussion regarding the similarities and differences between these two types of magnetic flux structures).

The original motivation for studying the SFFT was probably the desire to create novel active electronic applications for the new high-temperature superconductors, since good tunnel junction characteristics in high- $T_{c}$ materials are as yet difficult to realize (but see the paper by Alex Braginski [72] for recent progress on this topic); lately, however, the SFFT has begun to assume an increasing importance in its own right inasmuch as there are indications that it may display some performance characteristics that are superior to its long-junction counterparts. An indicative example is the SFFT amplifier described by Martens et al. [73] at 1992 ASC: this device showed a gain of $7 \mathrm{~dB}$ over a bandwidth of $50 \mathrm{GHz}$.

\section{Conclusions}

The central theme of the NATO ASI on which this present volume is based was that the time now seems ripe for the field of Superconducting Electronics, which has long played a Cinderella rôle, to make its debut at the ball. My task has been to present the notion that devices based on soliton propagation effects, which have been considered a particularly esoteric aspect of a generally esoteric field, deserve serious attention in this context. I have tried to show that the basic ideas involved are, in fact, quite readily accessible, and that they do offer a rich potential for practical applications. Here, I have focused on just two fairly simple analog applications - oscillators and amplifiers - leaving the exciting area of digital applications to the able exposition of Kostya Likharev [74]. If I succeed in communicating to the reader even a part of the enthusiasm that was prevalent during the ASI, then my efforts will have been amply repaid.

\section{Acknowledgements}

The work described herein is largely the fruit of a long-standing and felicitous collaboration between the Josephson junction groups working at the University of Salerno, the Cybernetics Institute of the Italian CNR, and the Technical University of Denmark; I wish to express my gratitude to my many friends and colleagues working in these groups. Special thanks go to Valery Koshelets, Jim Nordman, and Yongming Zhang for sending me prepublication material regarding their work on flux flow devices, and to Jim Nordman, Boris Malomed, Niels Pedersen, and Alexey Ustinov for illumi- 
nating comments and a critical reading of the manuscript. Financial support from MURST and from the Progetto Finalizzato "Tecnologie Superconduttive e Criogeniche" del CNR (Italy) is gratefully acknowledged.

\section{References}

[1] N. J. Zabusky and M. D. Kruskal, Phys. Rev. Lett. $\underline{15}$ (1965) 240.

[2] B. D. Josephson, Phys. Lett. 1 (1962) 251.

[3] I. O. Kulik, Zh. Eksp. Teor. Fiz. $\underline{51}$ (1966) 1952; Sov. Phys. JETP 24 (1967) 1307.

[4] A. C. Scott, Am. J. Phys. 37 (1969) 52.

[5] T. A. Fulton and R. C. Dynes, Solid State Comm. 12 (1973) 57.

[6] A. C. Scott, F. Y. F. Chu, and D.W. McLaughlin, Proc. IEEE $\underline{61}$ (1973) 1443.

[7] T. A. Fulton, R. C. Dynes, and P. W. Anderson, Proc. IEEE 61 (1973) 28.

[8] R. D. Parmentier, in Solitons in Action, K. Lonngren and A. Scott, eds. (Academic Press, New York, 1978), pp. 173-199.

[9] N. F. Pedersen, in Solitons, S. E. Trullinger, V. E. Zakharov, and V. L. Pokrovsky, eds. (Elsevier, Amsterdam, 1986), pp. 469-501.

[10] Y. Bruynseraede, C. Van Haesendonck, and V. V. Moshchalkov, this volume.

[11] A. Barone and G. Paternò, Physics and Applications of the Josephson Effect (Wiley, New York, 1982).

[12] J. C. Eilbeck, P. S. Lomdahl, O. H. Olsen, and M. R. Samuelsen, J. Appl. Phys. $\underline{57}$ (1985) 861.

[13] M. Scheuermann and C. C. Chi, in SQUID '85, H. D. Hahlbohm and H. Lübbig, eds. (de Gruyter, Berlin, 1985), pp. 477-481.

[14] A. C. Scott, Solid-State Electronics 7 (1964) 137.

[15] N. Grønbech-Jensen, Phys. Rev. B $\underline{45}$ (1992) 7315.

[16] T. A. Fulton, in Superconductor Applications: SQUIDs and Machines, B. B. Schwartz and S. Foner, eds. (Plenum, New York, 1977), pp. 125-187.

[17] M. Cirillo, R. D. Parmentier, and B. Savo, Physica D $\underline{3}$ (1981) 565.

[18] G. Costabile, R. D. Parmentier, B. Savo, D. W. McLaughlin, and A. C. Scott, Appl. Phys. Lett. 32 (1978) 587.

[19] G. Costabile, S. Pagano, and R. D. Parmentier, Phys. Rev. B $\underline{36}$ (1987) 5225.

[20] S. N. Erné, A. Ferrigno, and R. D. Parmentier, Phys. Rev. B $\underline{27}$ (1983) 5440.

[21] Y. Zhang, "Theoretical and Experimental Studies of the Flux-flow Type Josephson Oscillator", Licentiate thesis, Dept. Physics, Chalmers Univ. Tech., Göteborg, Sweden, 1991 (unpublished).

[22] K. Yoshida and F. Irie, in Low Temperature Physics-LT14, vol. 4, M. Krusius and M. Vuorio, eds. (North-Holland, Amsterdam, 1975), pp. 176-179.

[23] C. S. Owen and D. J. Scalapino, Phys. Rev. 164 (1967) 538.

[24] N. Grønbech-Jensen, P. S. Lomdahl, and M. R. Samuelsen, Phys. Rev. B $\underline{43}$ (1991) 12799.

[25] A. V. Ustinov, T. Doderer, R. P. Huebener, N. F. Pedersen, B. Mayer, and V. A. Oboznov, Phys. Rev. Lett., $\underline{69}$ (1992) 1815.

[26] D. W. McLaughlin and A. C. Scott, Phys. Rev. A 18 (1978) 1652.

[27] Yu. S. Kivshar and B. A. Malomed, Rev. Mod. Phys. 61 (1989) 763.

[28] A. Davidson, N. F. Pedersen, and S. Pagano, Appl. Phys. Lett. 48 (1986) 1306; see also: S. Pagano, Licentiate thesis, DCAMM Report S-42, Tech. Univ. Denmark, Lyngby, Denmark, 1987 (unpublished).

[29] M. Salerno, M. R. Samuelsen, G. Filatrella, S. Pagano, and R. D. Parmentier, Phys. Rev. B 41 (1990) 6641.

[30] G. Filatrella, G. Rotoli, and R. D. Parmentier, Phys. Lett. A 148 (1990) 122.

[31] O. A. Levring, N. F. Pedersen, and M. R. Samuelsen, J. Appl. Phys. 54 (1983) 987.

[32] N. F. Pedersen and A. Davidson, Phys. Rev. B $\underline{41}$ (1990) 178. 
[33] N. Grønbech-Jensen, "Coupling of microwaves to fluxon motion in Josephson junctions", Phys. Rev. B, in press.

[34] L. E. Guerrero and M. Octavio, Phys. Rev. A $\underline{40}$ (1989) 3371.

[35] M. P. Soerensen, N. Arley, P. L. Christiansen, R. D. Parmentier, and O. Skovgaard, Phys. Rev. Lett. $\underline{51}$ (1983) 1919.

[36] W. J. Yeh, O. G. Symko, and D. J. Zheng, Phys. Rev. B 42 (1990) 4080.

[37] G. Filatrella, R. D. Parmentier, S. Pagano, P. L. Christiansen, M. P. Sørensen, and N. Grønbech-Jensen, "On the switching between soliton dynamic states in long Josephson junctions", Phys. Lett. A, in press.

[38] B. A. Malomed, Phys. Rev. B $\underline{41}$ (1990) 2037.

[39] G. Rotoli and G. Filatrella, Phys. Lett. A 156 (1990) 211; G. Rotoli and G. Filatrella, in Nonlinear Coherent Structures in Physics and Biology, M. Remoissenet and M. Peyrard, eds. (Springer-Verlag, Berlin, 1991), pp. 284-291.

[40] See, e.g.: E. Ott, Rev. Mod. Phys. 53 (1981) 655.

[41] M. Salerno, Phys. Rev. B $\underline{44}$ (1991) 2720.

[42] G. Filatrella, G. Rotoli, and M. Salerno, "Chaos suppression in fluxon dynamics of long Josephson junctions", preprint, 1992.

[43] E. Joergensen, V. P. Koshelets, R. Monaco, J. Mygind, M. R. Samuelsen, and M. Salerno, Phys. Rev. Lett. 49 (1982) 1093.

[44] M. Cirillo, I. Modena, P. Carelli, G. S. Spagnolo, R. Leoni, and M. Pullano, IEEE Trans. Magn. 27 (1991) 3335.

[45] T. Nagatsuma, K. Enpuku, F. Irie, and K. Yoshida, J. Appl. Phys. $\underline{54}$ (1983) 3302.

[46] S. Kiryu, S. Kohjiro, A. Shoji, S. Kodaira, J. Itanani, and S. Sato, 1992 Applied Superconductivity Conference, Chicago, U.S.A., August 1992, paper ELD-9.

[47] J. E. Lukens, in Modern Superconducting Devices, S. T. Ruggiero and D. A. Rudman, eds. (Academic Press, New York, 1990), pp. 135- .

[48] M. Cirillo and F. L. Lloyd, J. Appl. Phys. 61 (1987) 2581.

[49] S. Pagano, R. Monaco, and G. Costabile, IEEE Trans. Magn. 25 (1989) 1080.

[50] R. Monaco, N. Grønbech-Jensen, and R. D. Parmentier, Phys. Lett. A 151 (1990) 195.

[51] P. Barbara, A. Davidson, G. Filatrella, J. Holm, J. Mygind, and N. F. Pedersen, Phys. Lett. A $\underline{165}(1992) 241$.

[52] A. V. Ustinov,J. Mygind, and V. A. Oboznov, J. Appl. Phys. $\underline{72}$ (1992) 1203.

[53] G. Filatrella, G. Rotoli, N. Grønbech-Jensen, R. D. Parmentier, and N. F. Pedersen, J. Appl. Phys. $\underline{72}$ (1992) 3179.

[54] D.-G. Crete, P. Feautrier, M. Hanus, R. Monaco, and P. J. Encrenaz, in Stimulated Effects in Josephson Devices, M. Russo and G. Costabile, eds. (World Scientific, Singapore, 1990), pp. 87-98.

[55] Y. M. Zhang, D. Winkler, and T. Claeson, 1992 Applied Superconductivity Conference, Chicago, U.S.A., August 1992, paper ELD-7.

[56] Y. M. Zhang, D. Winkler, and T. Claeson, "Linewidth measurements of Josephson flux-flow oscillators in the band 280-330 GHz", preprint, 1992.

[57] V. P. Koshelets, A. V. Shchukin, S. V. Shitov, and L. V. Filippenko, 1992 Applied Superconductivity Conference, Chicago, U.S.A., August 1992, paper ELD-8.

[58] A. D. Smith, this volume.

[59] T. V. Rajeevakumar, Appl. Phys. Lett. 39 (1981) 439.

[60] D. P. McGinnis, J. B. Beyer, and J. E. Nordman, J. Appl. Phys. 59 (1986) 3917.

[61] K. K. Likharev, V. K. Semenov, O. V. Snigirev, and B. N. Todorov, IEEE Trans. Magn. 15 (1979) 420.

[62] B. J. Van Zeghbroeck, Appl. Phys. Lett. $\underline{42}$ (1983) 736.

[63] D. P. McGinnis, J. B. Beyer, and J. E. Nordman, IEEE Trans. Magn. 25 (1989) 1262.

[64] T. Hashimoto, K. Enpuku, K. Yoshida, and K. Yamafuji, Japan. J. Appl. Phys. 26 (1987) L1874.

[65] I. P. Nevirkovets, E. M. Rudenko, and A. V. Ustinov, Fiz. Nizk. Temp. 14 (1988) 1134. 
[66] A. Petraglia, "Non Linear Dynamics in Long Josephson Junctions", Graduation thesis, Dept. Physics, Univ. Salerno, Salerno, Italy, 1992 (unpublished).

[67] K. K. Likharev, Sov. Phys. JETP 34 (1972) 906.

[68] J. S. Martens, D. S. Ginley, J. B. Beyer, J. E. Nordman, and G. K. G. Hohenwarter, IEEE Trans. Appl. Supercon. 1 (1991) 95.

[69] J. S. Martens, V. M. Hietala, T. E. Zipperian, D. S. Ginley, and C. P. Tigges, in Superconducting Devices and Their Applications, H. Koch and H. Lübbig, eds. (Springer-Verlag, Berlin, 1992), pp. 186-191.

[70] S. Hontsu and J. Ishii, J. Appl. Phys. 63 (1988) 2021.

[71] K. K. Likharev, Rev. Mod. Phys. $\underline{51}(1979) 101$.

[72] A. I. Braginski, this volume.

[73] J. S. Martens, V. M. Hietala, T. A. Plut, D. S. Ginley, G. A. Vawter, C. P. Tigges, M. P. Siegal, J. M. Phillips, and S. Y. Hou, 1992 Applied Superconductivity Conference, Chicago, U.S.A., August 1992, paper EJ-2.

[74] K. K. Likharev, this volume. 